\title{
Intentional Death Caused by the Overdose of Nonprescription Drugs: An Autopsy Case of Acetaminophen Poisoning
}

Milim Kim ${ }^{1,2}$, Soong Deok Lee ${ }^{3,4}$, Moon-Young $\mathrm{Kim}^{4}$

${ }^{1}$ Department of Pathology, Seoul National University Bundang Hospital, Seongnam, Korea, ${ }^{2}$ Department of Pathology, Seoul National University College of Medicine, Seoul, Korea, ${ }^{3}$ Department of Forensic Medicine, Seoul National University College of Medicine, Seoul, Korea, ${ }^{4}$ Institute of Forensic and Anthropological Science, Seoul National University College of Medicine, Seoul, Korea

Received: April 23, 2020

Revised: May 14, 2020

Accepted: May 19, 2020

\section{Correspondence to}

Moon-Young Kim Institute of Forensic and Anthropological Science, Seoul National University College of Medicine, 103 Daehak-ro, Jongno-gu, Seoul 03080, Korea

Tel: +82-2-740-8353

Fax: +82-2-764-8340

E-mail: tttrack@naver.com
Acetaminophen or paracetamol ( $\mathrm{N}$-acetyl-para-aminophenol [APAP]) is a safe and effective antipyretic and analgesic drug and is a representative nonprescription drug. However, APAP is one of the most common nonprescription drugs used for intentional overdose or suicide, thereby resulting in hundreds of deaths annually in the United States. Moreover, the misuse of nonprescription drugs is a cause of increasing concern in Korea with the revision of the Pharmaceutical Affairs Law in 2012. Generally, the mortality rate of APAP overdose is extremely low due to the well-established treatment guidelines and availability of antidotes. However, it should not be overlooked because of the high number of either accidental or intentional APAP overdose cases recorded every day. To achieve a good prognosis, individuals with APAP overdose must be immediately identified and brought to the hospital. Herein, we report an autopsy case of an individual with APAP overdose who died due to acute liver injury.

Key Words: Nonprescription drugs; Acetaminophen; Drug-induced acute liver injury; Suicide; KIDS KAERS database (KIDS-KD)

\section{Introduction}

Acetaminophen or paracetamol ( $\mathrm{N}$-acetyl-paraaminophenol [APAP]) is an effective antipyretic and mild analgesic drug and is widely used as a representative nonprescription medication worldwide. Although there is a general perception that the use of APAP is safe for the public, it can be fatal as either accidental or intentional overdose can cause hepatotoxicity. APAP overdose accounts for $8.8 \%(276 / 3,125)$ of all cases of fatalities due to poisoning in the United States in 2018 [1]. In addition, it is the most common cause of acute liver failure not only in the United States but also in several European countries, including Great Britain [2]. 
In Korea, several clinicians encounter patients with APAP overdose every day in practice. The mortality rate of APAP poisoning is extremely low owing to the well-established treatment guidelines and availability of effective antidotes. However, the actual rate of prevalence and mortality due to APAP poisoning is not known in Korea [3,4]. Few studies have reported about intentional APAP overdose in Korea. However, fatal cases seem to be extremely rare [5-11]. The revised Pharmaceutical Affairs Law in Korea allowed access to 13 nonprescription medications, including APAP, at retailers and convenience stores in November 2012. Thus, the risk of an intentional overdose of these drugs has significantly increased due to easier access than before. A previous study in Korea has shown that the risk is higher in adolescents who have difficulties obtaining prescription drugs by themselves [6].

In relation to this context, the information that APAP overdose can be fatal should be provided to increase knowledge among healthcare workers, policymakers, and even the public. Herein, we report an autopsy case of an individual with intentional APAP overdose who died due to acute liver injury.

\section{Case Report}

A 46-year-old man was found dead in his home by his mother. After the last call 10 days prior, the man did not answer his phone, which prompted his mother to visit him. She claimed that the gate was locked with no evidence of trespassing. During the inspection, white powders were found in the perioral and intraoral areas of the deceased and also scattered on his neck (Fig. 1), his clothes, the floor, and the sink of the kitchen. Similar powder residues were observed in the blender, and several empty Tyrenol boxes were found in a plastic bag near the blender. The police found a summon from the court and a handwritten will by the man inside the house.

During autopsy, the man's body was in the early stage of decomposition. There was no evidence of external force applied. The white powder was not found grossly in his liquefied stomach content $(30 \mathrm{~mL})$. However, there were multiple hemorrhagic spots in the gastric mucosa (Fig. 2A). His liver weighed $1,035 \mathrm{~g}$, and fatty changes were noted grossly (Fig. 2B). There was no specific

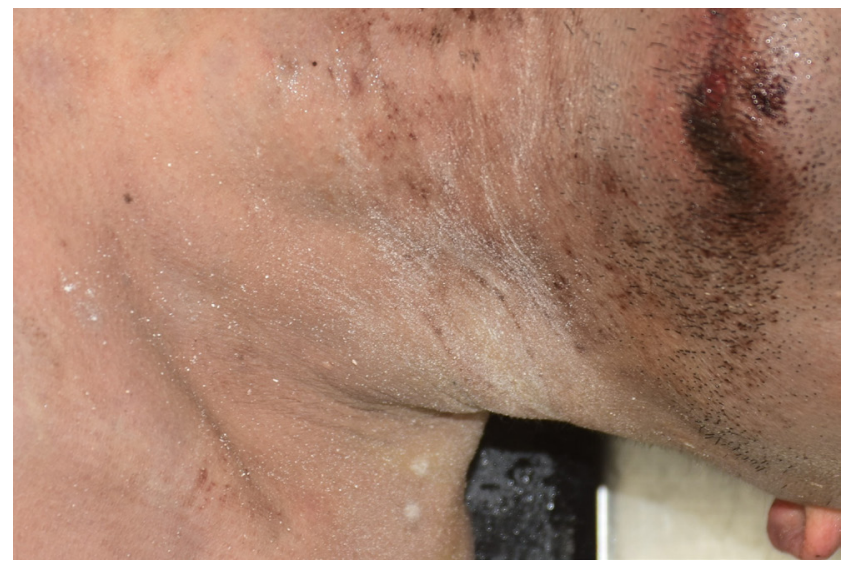

Fig. 1. White powder shed around the neck of the deceased.

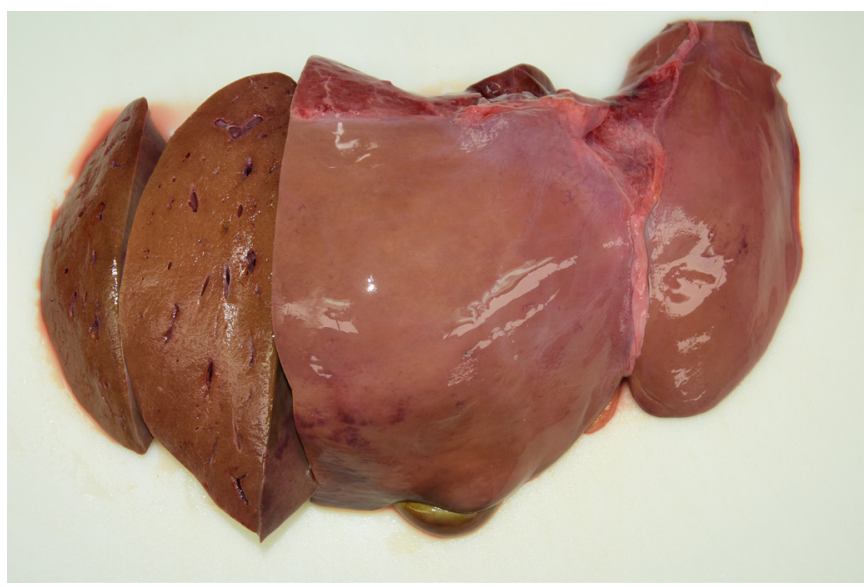

B

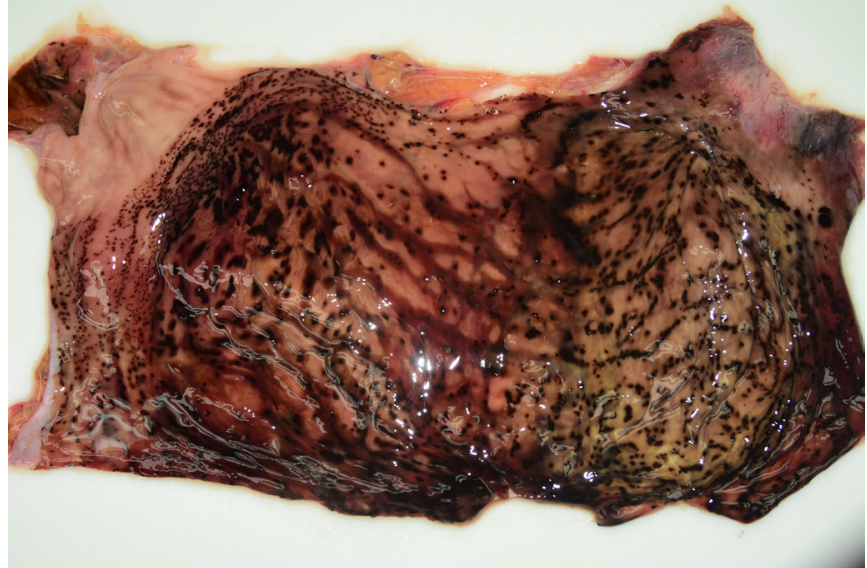

Fig. 2. Hemorrhagic spots of the gastric mucosa (A) and fatty change of liver (B) in the gross examination. 
finding in other organs. Microscopic examination revealed hepatocyte damage of centrilobular pattern, and the portal area was relatively preserved (Fig. 3A). Apoptotic hepatocytes with pyknotic bodies were observed in this area with ballooning degeneration and bile pigments (Fig. 3B). Based on the toxicology findings, APAP and chlorpheniramine were detected in the blood in his heart and the gastric content. The concentration of APAP in the blood was $1,943 \mathrm{mg} / \mathrm{L}$, which was significantly higher than the toxic or lethal level in general. That of chlorpheniramine was within the therapeutic range. According to these results, the cause and mechanism of death were APAP poisoning and consequent acute liver injury, respectively.

\section{Discussion}

According to the United States Food and Drug Administration, APAP is generally considered safe, and it is used as a nonprescription drug in most countries, with some exceptions according to dose, dosage form, and combined ingredients. However, even though the drug is popular, hundreds of deaths due to APAP poisoning are recorded in the United States annually. Thus far, mortality due to poisoning from APAP or other nonprescription drugs has not been reported in Korea.

Hepatotoxicity and liver failure are the main mechanisms of APAP poisoning. APAP is rapidly absorbed in the duodenum. The majority of ingested APAP are inactivated by sulfation and glucuronidation and are then excreted in the urine. The rest are transformed to $\mathrm{N}$-acetyl-para-benzoquinone imine (NAPQI), a highly reactive toxic metabolite, by cytochrome $\mathrm{P} 450$ enzymes (CYP450) in the liver [12]. When overdose occurs, the capacity for sulfation and glucuronidation becomes saturated, leading to the excessive production and accumulation of NAPQI in hepatocytes. NAPQI depletes the glutathione capacity of hepatocytes, which induces mitochondrial dysfunction, and produces covalent bonds with critical cellular proteins, which disturbs the cell metabolism. These mechanisms result in hepatotoxicity in patients with APAP overdose [13,14]. The guidelines on the management of APAP poisoning recommend a full course of antidote therapy for all ingestions $>10 \mathrm{~g}$ or $200 \mathrm{mg} / \mathrm{kg}[3,4]$. Based on the serum APAP concentration, patients with $>150 \mathrm{mg} / \mathrm{L} 4$ hours after ingestion should be treated according to the RumackMatthew nomogram, a graph used for the treatment of acetylcysteine therapy against APAP poisoning [4]. Within therapeutic doses of up to $4,000 \mathrm{mg}$ every 24 hours, the serum concentration of APAP is not expected to exceed $20 \mathrm{mg} / \mathrm{L}[4]$. The APAP concentration of the deceased was $1,943 \mathrm{mg} / \mathrm{L}$, which was higher than these figures.

The clinical presentation of APAP overdose are classified based on the time interval after ingestion

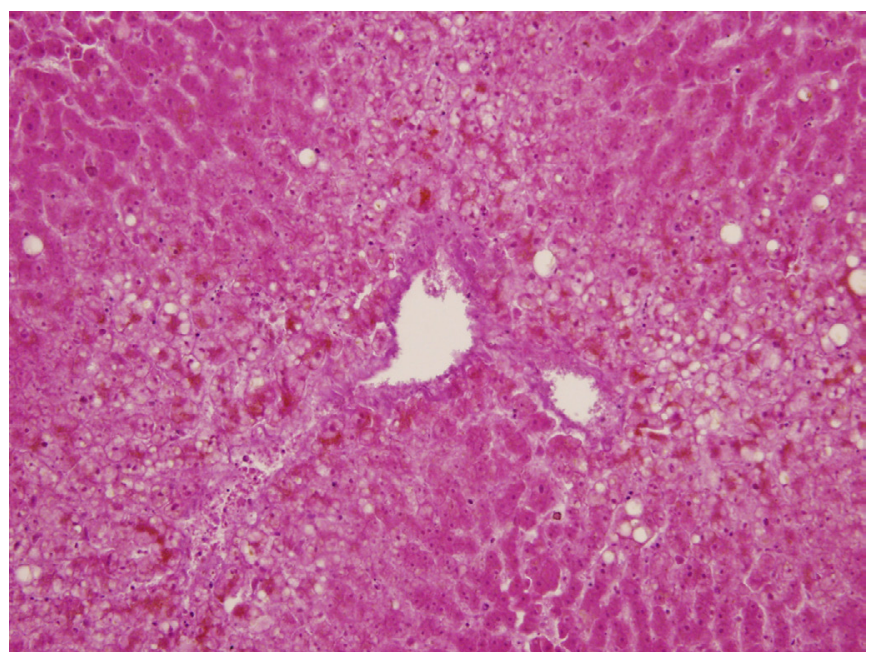

A

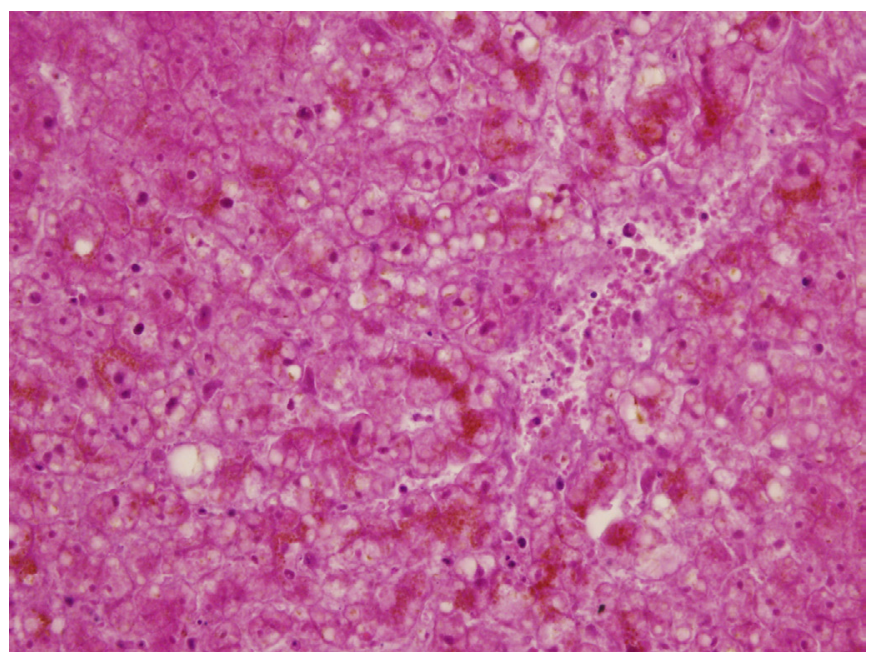

Fig. 3. Microscopic findings of the liver showing centrilobular necrosis $(A, H \& E, \times 200)$ with balloon degeneration and pyknotic bodies $(B, H \& E$, $\times 400)$. 
Table 1. Suspected cases of intentional APAP overdose searched from KIDS-KD from 2008 to 2017

\begin{tabular}{cccccccc}
\hline Age $(\mathrm{yr})$ & Sex & Underlying disease & Purpose & Dose & Symptom or sign & Combined drug & Result \\
\hline 20 & $\mathrm{M}$ & Depression & Pain relieve & 100 tablets & Nausea, liver dysfunction & None & Recovery \\
42 & $\mathrm{~F}$ & Tension headache & Suicide & 14 tablets & Dizziness, liver enzyme increase & None & Recovery \\
28 & $\mathrm{~F}$ & Depression & Unknown & $20.8 \mathrm{~g}$ & Loss of consciousness & Alprazolam (8 mg) & Recovery \\
17 & $\mathrm{~F}$ & Unknown & Suicide & 18 tablets & Abdominal discomfort & None & Unknown \\
Unknown & $\mathrm{F}$ & Unknown & Unknown & $13 \mathrm{~g}$ & Acute liver injury & None & Unknown \\
\hline
\end{tabular}

APAP, N-acetyl-para-aminophenol; KIDS-KD, KIDS KAERS database.

[13,15]. During stage 1 (within the first 24 hours after ingestion), individuals may experience abdominal pain, vomiting, lethargy, malaise, and diaphoresis. These symptoms diminish during stage 2 (24-72 hours), and the biochemical abnormalities become evident, with elevated transaminase and bilirubin levels and prolonged prothrombin time. In stage 3 (72-96 hours), central nervous system symptoms, including confusion, may be observed with the reappearance of nausea and vomiting. Multi-organ failure, including acute liver and renal failure, can occur, which may lead to death. Microscopic hepatocellular injury and apoptosis are commonly observed in this stage. The centrilobular zone $I$ is commonly affected because CYP450 is mainly distributed in this area. However, with an extremely high APAP dose, necrosis could extend to zone II [16]. The recovery of liver damage starts in stage 4 (4-14 days), and the structure of the liver parenchyma may return to normal within 3 months. Regardless of these courses, the patient may die within a few days if there is no adequate therapeutic intervention, which might be the case of the deceased in this report [17].

A single-center study reported that 108 patients had visited their emergency department due to APAP overdose within 3 years. In another study, 321 patients were diagnosed APAP overdose within 17 years. However, mortality was not clearly mentioned in these studies [5,7]. Another study reported that there were 125 patients with APAP or nonsteroidal antiinflammatory drugs overdose with 14 deaths within 9 years. However, the causative substance was not specified [10]. According to the information in the Korea Institute of Drug Safety and Risk Management-Korea Adverse Event Reporting System Database (KIDS-KD), a national pharmacovigilance database established based on the Korea Adverse Event Reporting System of KIDS, 2,832 cases of APAP administration were recorded from 2008 to 2017 as related to severe adverse drug reaction correlated to acute cardiac, respiratory, hepatic, and neurologic dysfunction, suicidal attempt, or death [11]. After thorough exclusions, five cases were suspected as intentional APAP overdose due to significantly high doses and transient use. However, there was no mortality recorded (Table 1). These data indicate that there is a high number of patients with APAP overdose in Korea but the mortality rate is extremely low as patients are treated in medical institutions. This phenomenon may be attributed to the well-established treatment guidelines of APAP overdose and high accessibility and good quality of medicines in Korea. Nevertheless, APAP overdose might be life-threatening if a patient is not brought to a hospital for treatment at an earlier time. With the progression of social individualization, as represented by an increase in single-person households and individuals dying alone in all generations in Korea, more efforts should be exerted to actively identify and monitor patients who may have suicidal ideations. In this study, the deceased was living alone and did not frequently keep in touch with his family or friends, and these factors seem to have prevented him to be detected early after the ingestion of APAP.

If a patient with APAP poisoning is not treated in a medical institution and is found dead as this case, the body should be evaluated as an unnatural death of unknown cause, and an autopsy should be conducted. Thus far, there are no official databases or systematically analyzed reports about death due to poisoning based on autopsy results in Korea. Only one study had analyzed death due to poisoning in a single province. However, the detailed characteristics of the poisonous 
substances were not discussed [18]. In Toronto, Canada, the rate of mortality due to nonprescription drugs accounts for about $21.5 \%$ of all cases of suicides due to overdose, and the most commonly used drug was diphenhydramine $(\sim 14.5 \%)$, an antihistamine used for the management of insomnia, followed by APAP ( $4.5 \%)$ and salicylates ( $3.0 \%)$, with a slight overlap between each other due to combined use [19]. A report about young individuals who attempted suicide in Korea showed that the three leading drugs used for suicide were acetaminophen-containing analgesics (42.9\%, 12/28), antihistamines (e.g., diphenhydramine, doxylamine), and aspirin (21.4\%, 6/28, respectively) [8]. This finding is similar to cases in Toronto. They showed that high accessibility to nonprescription drugs (including financial and geographical aspects) may be critical when selecting the substances for suicide among children and adolescents. This factor would be also important to adults. The public concern about the revision of the Pharmaceutical Affairs Act in 2012 that this action may encourage intentional overdoses of nonprescription drugs due to easier accessibility has not become reality yet [10]. However, this should be assessed continuously as various media platforms, which include not only traditional ones, such as newspaper and television reports, but also more personal ones, such as the internet and social networking services, have suicide-promoting influences.

Several strategies that can prevent the intentional overdose of nonprescription drugs, including nationwide statistical research about the pattern of intentional overdose in Korea, use of a surveillance system for unusual large purchasing, modification of packages or dosage forms to ensure safety, and providing education for public and medical workers about the proper use, could be applied. The identification of the types and characteristics of nonprescription drugs that are commonly misused in Korea is beneficial in preventing deaths due to intentional overdose as in this case. However, these strategies must aim to identify patients with suicidal ideations and properly treat and support them using the social system. If not, these individuals may choose another method of suicide [19]. To the best of our knowledge, this is the first autopsy case of APAP poisoning in Korea. Thus, this study can emphasize the misuse of nonprescription drugs among individuals with suicidal ideations in Korea. The experience and knowledge during the establishment of the death investigation system could provide basic data for the prevention of intentional overdose of nonprescription drugs.

ORCID: Milim Kim: https://orcid.org/0000-0001-69661606; Soong Deok Lee: https://orcid.org/0000-00034270-4279; Moon-Young Kim: https://orcid.org/00000002-6381-9377

Conflicts of Interest

No potential conflict of interest relevant to this article was reported.

\section{References}

1. Gummin DD, Mowry JB, Spyker DA, et al. 2018 Annual Report of the American Association of Poison Control Centers' National Poison Data System (NPDS): 36th Annual Report. Clin Toxicol (Phila) 2019;57:1220-413.

2. Ostapowicz G, Fontana RJ, Schiodt FV, et al. Results of a prospective study of acute liver failure at 17 tertiary care centers in the United States. Ann Intern Med 2002;137:947-54.

3. Chung SP, Kim SH, Lee HS. Acetaminophen poisoning. J Korean Soc Clin Toxicol 2008;6:1-8.

4. Chiew AL, Reith D, Pomerleau A, et al. Updated guidelines for the management of paracetamol poisoning in Australia and New Zealand. Med J Aust 2020;212:175-83.

5. Bum JH, Rhee N, Kim MJ, et al. Extended blood drug concentrations in extended release formulated acetaminophen overdose patients.J Korean Soc Clin Toxicol 2011;9:71-6.

6. Lee OS, Cheon YJ, Kim JT, et al. Evaluation of drugs for suicide attempt and antidote uses in emergency room of a hospital in Korea. Korean J Clin Pharm 2012;22:304-15.

7. Chae HJ, Rhee NG, Kim HJ, et al. Oral vs. intravenous administration of $\mathrm{N}$-acetylcysteine in the acetaminophen poisoning.J Korean Soc Clin Toxicol 2012;10:97-102.

8. Kweon YS, Hwang S, Yeon B, et al. Characteristics of drug overdose in young suicide attempters. Clin Psychopharmacol Neurosci 2012;10:180-4.

9. Kim JH, Jeong WJ, Ryu R, et al. Is it adequate to determine acetaminophen toxicity solely on patients' history? An analysis on clinical manifestation of intoxication patients with positive serum acetaminophen concentrations. J Korean Soc Clin Toxicol 2017;15:94-100.

10. Kim CY, Lee EJ, Lee SW, et al. Changes in toxicological characteristics after sales of nonprescription drugs in convenience stores.J Korean Soc Clin Toxicol 2018;16:42-8. 
11. KIDS-KAERS database (KIDS-KD) [Internet]. Anyang: Korea Institute of Drug Safety \& Risk Management; 2008-2017 [cited 2019 May 17]. Available from: https://www.drugsafe.or.kr/.

12. McGill MR, Jaeschke H. Metabolism and disposition of acetaminophen: recent advances in relation to hepatotoxicity and diagnosis. Pharm Res 2013;30:2174-87.

13. Chun $\sqcup$, Tong MJ, Busuttil RW, et al. Acetaminophen hepatotoxicity and acute liver failure.J Clin Gastroenterol 2009;43:342-9.

14. Yuan L, Kaplowitz N. Mechanisms of drug-induced liver injury. Clin Liver Dis 2013;17:507-18.

15. Yoon E, Babar A, Choudhary M, et al. Acetaminophen-induced hepatotoxicity: a comprehensive update. J Clin Transl Hepatol
2016;4:131-42.

16. Ghanem $\mathrm{Cl}$, Perez MJ, Manautou JE, et al. Acetaminophen from liver to brain: new insights into drug pharmacological action and toxicity. Pharmacol Res 2016;109:119-31.

17. Larson AM. Acetaminophen hepatotoxicity. Clin Liver Dis 2007;11:525-48.

18. Choi WY, Kim HJ, Na JY, et al. Analysis of death due to poisoning in Gwangju and Jeollanam-do areas. Korean J Leg Med 2016;40:72-7.

19. Sinyor $M$, Howlett $A$, Cheung $A H$, et al. Substances used in completed suicide by overdose in Toronto: an observational study of coroner's data. Can J Psychiatry 2012;57:184-91. 